\title{
Performance Evaluation of Wlan by Varying Pcf, Def and Enhanced Def Slots To Improve Quality of Service
}

\author{
Inderjeet Kaur ${ }^{1}$, Manju Bala ${ }^{2}$, Harpreet Bajaj ${ }^{3}$ \\ ${ }^{1,3}$ (Computer Science \& Engg., DAVIET /PTU, India) \\ 2 (Information Technology, DAVIET/PTU, India)
}

\begin{abstract}
Researchers have proposed a number of co-ordination functions in literature for improving quality of service. Each one is based on different characteristics and properties. In this paper, we evaluate the performance of wireless network using PCF, DCF \& EDCF. We perform simulations using OPNET IT GURU Academic Edition simulator. In the performance evaluation of the co-ordination function, the protocols are tested under the realistic conditions. Tests are performed for various parameters (Delay, Data Dropped, Throughput) in wireless Networks. This OPNET simulation shows the impact of co-ordination function for wireless networks for different parameters.
\end{abstract}

Keywords - Data Dropped, Delay, DCF, EDCF, PCF, Throughput, Traffic Sent, Traffic Received.

\section{INTRODUCTION}

WLAN applications are mainly data centric; there is growing demand for real time services over WLAN [1]. IEEE 802.11 is the most popular standard used in Wireless Local Area Networks (WLANs). The IEEE 802.11 standard has defined two different access mechanisms in order to allow multiple users to access a common channel, the distributed coordination function (DCF) and a centrally controlled access mechanism called the point coordination function (PCF) [2]. The following three basic access mechanisms have been defined for IEEE 802.11: the mandatory basic access method based on CSMA/CA, an optional method avoiding hidden terminal problem, and finally a contention-free polling method for time bounded services. The first two methods are also summarized as Distributed Coordination Function (DCF); the third method is called Point Coordination Function (PCF) [3]. In DCF, data frames are transmitted via two mechanisms, i.e., basic access mechanism and request-to-send/clear-to- send (RTS/CTS) mechanism [5]. In Basic Access Mechanism, Channel remains idle for a period of time Distribution Inter Frame Space (DIFS). The Station generates random backoff interval. It employs discrete-time backoff scale. The Transmission takes at beginning of time slot. Backoff time is chosen between $(0, \mathrm{w}-1)$. Backoff time counter decremented to 0 .

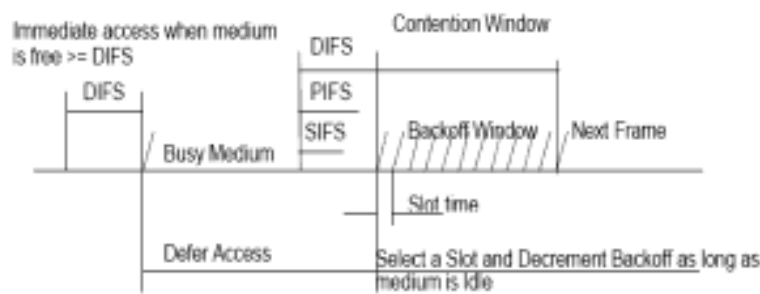

Fig 1: The Timing Relationship of DCF [7]

The RTS/CTS mechanism reserves the medium before transmitting a data frame by transmitting an RTS frame and replying with a CTS frame [6]. The original 802.11 MAC defines another coordination function called the Point Coordination Function (PCF): this is available only in "infrastructure" mode, where stations are connected to the network through an Access Point [4]. The PCF is located directly above the Distributed Coordination Function (DCF), in the IEEE 802.11 MAC Architecture. In this time is divided into contention period (CP) and contention free period (CFP). During CP, transfers used DCF, i.e., Data-ACK, or RTS-CTSData-ACK, with exponential back-off etc. During CFP, the AP controls all transmissions. That is, the AP controls which station transmits to the AP and which station receives packets from the AP. All stations can receive packets during the CFP. But the ability to transmit during the CFP is optional. During CFP all durations are set of $2^{\wedge} 15$, so station set their NAVs accordingly. The CFP ends with an explicit end of CFP frame from the AP. EDCF is designed to provide prioritized QoS by enhancing the contention-based DCF. It provides differentiated, distributed access to the wireless medium for QoS stations (QSTAs) using 8 different user priorities (UPs). 


\begin{tabular}{|c|c|c|}
\hline Priority & $\begin{array}{c}\text { Access } \\
\text { Category(AC) }\end{array}$ & Designation \\
\hline $\mathbf{1}$ & $\mathbf{0}$ & Background \\
\hline $\mathbf{2}$ & $\mathbf{0}$ & Standard \\
\hline $\mathbf{0}$ & $\mathbf{1}$ & Best Effort \\
\hline $\mathbf{3}$ & $\mathbf{1}$ & Excellent Effort \\
\hline $\mathbf{4}$ & $\mathbf{2}$ & Streaming Multimedia \\
\hline $\mathbf{5}$ & $\mathbf{2}$ & Interactive \\
\hline $\mathbf{6}$ & $\mathbf{3}$ & Interactive Voice \\
\hline $\mathbf{7}$ & $\mathbf{3}$ & Reserved \\
\hline
\end{tabular}

Table 1: User Priorities

Before entering the MAC layer, each data packet received from the higher layer is assigned a specific user priority value. The EDCF mechanism defines four different first-in first-out queues called access categories (ACs). Each data should be mapped into a corresponding AC Access Classes.

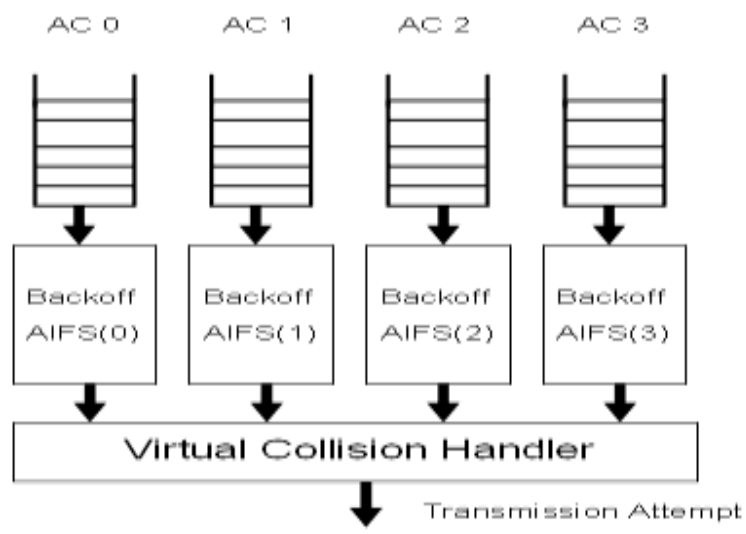

Fig 2: The Timing Relationship of EDCF [7]

After mentioning the introduction in section 1, our approach, scenarios and settings is discussed in section2. In section 3, we have described the result and discussion and finally in section 4, conclude the paper.

\section{APPROACH, SCENARIOS AND SETTINGS}

OPNET IT Guru Academic Edition is a utility designed with educational purposes in mind, specifically to help users be introduced to the domain of networking. The user can also develop projects by choosing a network scale, then choosing the model family and then making use of an object palette that includes items such as servers, routers, switches and others. Our scenario has eighteen wireless LAN-based workstations in a simple network configuration (Infrastructured BSS) which demonstrates the PCF access method used by the Wireless LAN. PCF provides a contention-free (CF) frame transfer. The medium access during the CF is regulated by the Point Coordinator (PC) which resides in the access point (AP). The traffic flows between the stations have been configured as,

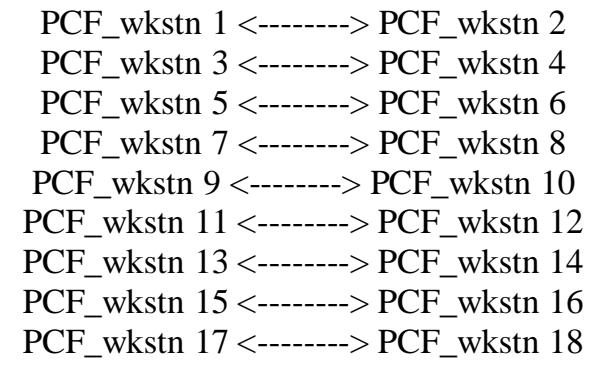


All the PCF related configuration parameters are grouped into a single compound attribute "PCF Parameters". The stations can participate in frame transfers during CF period by enabling the "PCF Functionality" attribute on the work stations.

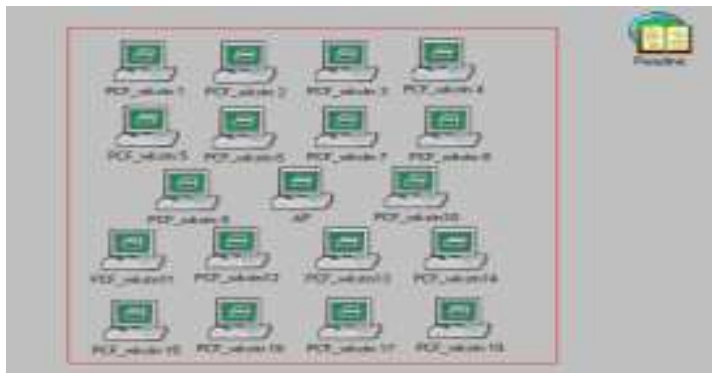

Fig 3: SCENARIO I

This scenario has eighteen wireless LAN-based workstations in a simple network configuration (Infrastructured BSS) which demonstrates the DCF access method used by the Wireless LAN. DCF provides a contention-Period (CP) frame transfer. The traffic flows between the stations have been configured as,
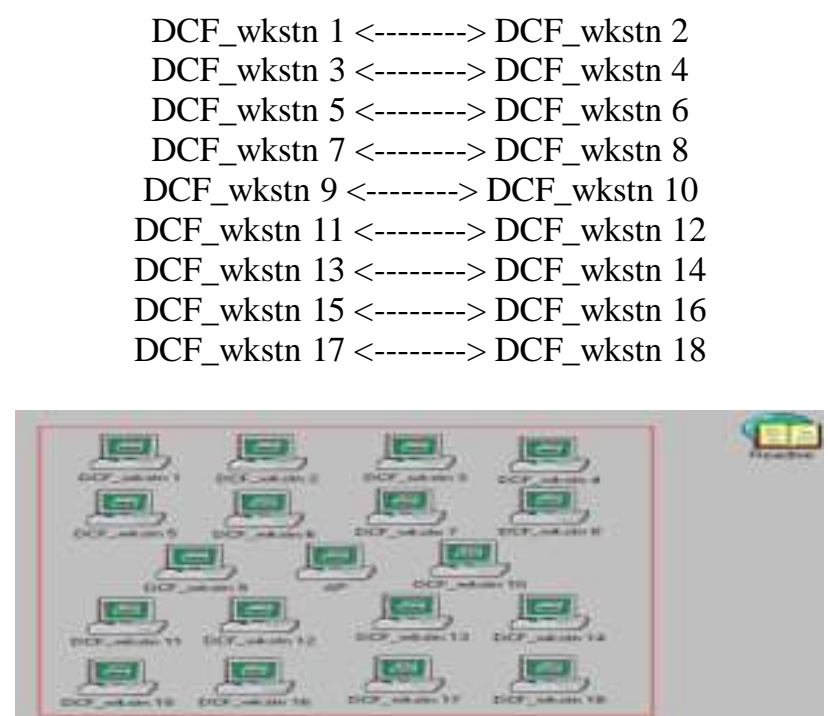

Fig 4: SCENARIO 2

This scenario has eighteen wireless LAN-based workstations in a simple network configuration (Infrastructured BSS) which demonstrates the EDCF access method used by the Wireless LAN. The traffic flows between the stations have been configured as,
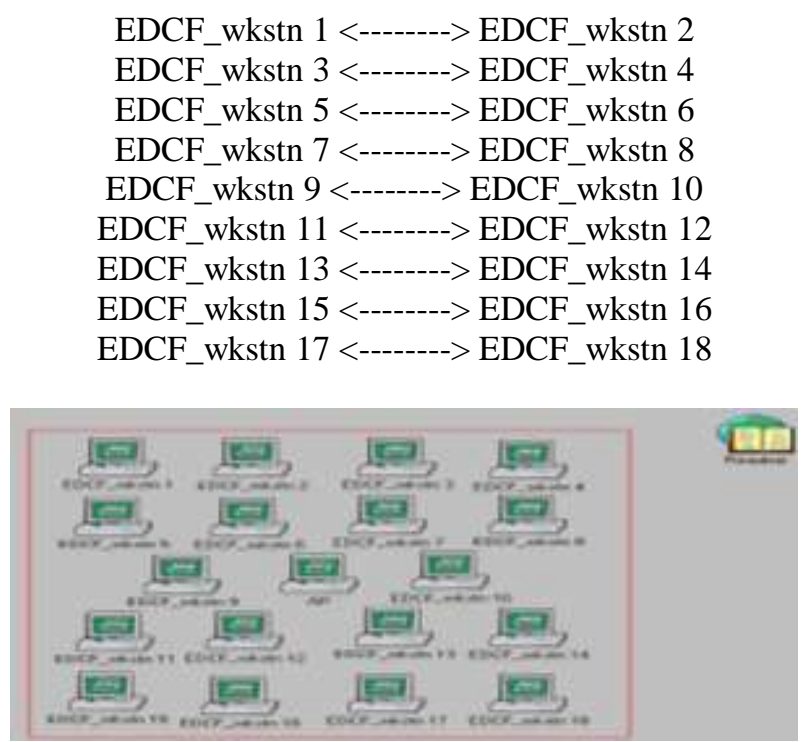

Fig 5: SCENARIO 3

www.iosrjournals.org 


\begin{tabular}{|c|c|l|}
\hline Application & Parameters & \multicolumn{1}{|c|}{ Units } \\
\hline \multirow{4}{*}{ WLAN } & Channel Reservation & Sec \\
\cline { 2 - 3 } & Data Traffic Received & Bits/Sec \\
\cline { 2 - 3 } & Data Traffic Sent & Bits/Sec \\
\cline { 2 - 3 } & Dropped Data Packet & Bits/Sec \\
\cline { 2 - 3 } & Retransmission Attempts & Packets \\
\cline { 2 - 3 } & Load & Bits/Sec \\
\hline
\end{tabular}

Table 2: Simulated Parameters

\section{SIMULATION EVALUATION \& RESULT ANALYSIS}

Six graphs are selected after simulating our models. All graphs show a combination of the three scenarios. In figure 6, it has been noticed that Channel reservation is better in case of EDCF. Channel reservation with EDCF varies from $33 \mathrm{~ms}$ to $34.7 \mathrm{~ms}$, with DCF and PCF it is $10 \mathrm{~ms}$. Therefore it is concluded that the results are better with EDCF.

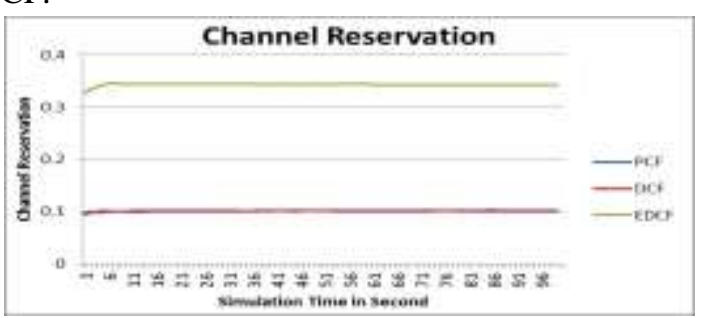

Fig 6: Average Wireless LAN Channel Reservation

In figure 7, it has been noticed that the traffic sent for PCF varies from $659 \mathrm{~kb} / \mathrm{sec}$ to $677 \mathrm{~kb} / \mathrm{sec}$, DCF $662 \mathrm{~kb} / \mathrm{sec}$ to $675 \mathrm{~kb} / \mathrm{sec}$ and EDCF $613 \mathrm{~kb} / \mathrm{sec}$ to $685 \mathrm{~kb} / \mathrm{sec}$. Therefore it is concluded that the results are better with EDCF.

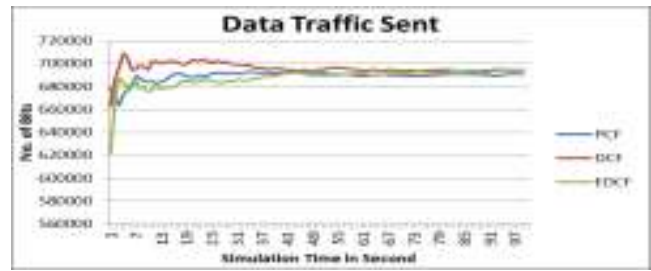

Fig 7: Average Wireless LAN Traffic Sent

In figure 8 , it has been noticed that the Traffic Received for PCF varies from $27 \mathrm{~kb} / \mathrm{sec}$ to $10 \mathrm{~kb} / \mathrm{sec}$, DCF $139 \mathrm{~kb} / \mathrm{sec}$ to $149 \mathrm{~kb} / \mathrm{sec}$ and EDCF $269 \mathrm{~kb} / \mathrm{sec}$ to $279 \mathrm{~kb} / \mathrm{sec}$. Therefore it is concluded that the results are better with EDCF.

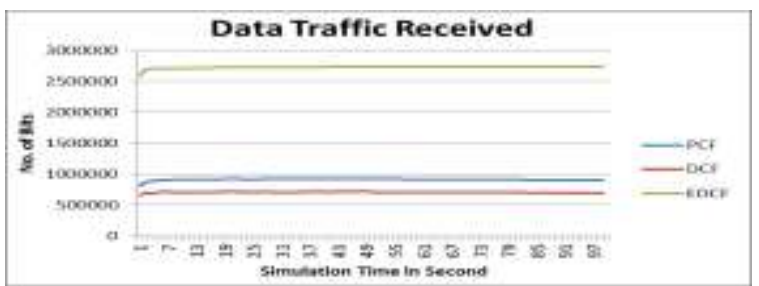

Fig 8: Average Wireless LAN Traffic Received

In figure 9, it has been noticed that the Data Dropped for PCF varies from 0 bits $/ \mathrm{sec}$ to $2761 \mathrm{bits} / \mathrm{sec}$, DCF 0bits/sec to 625bits/sec and with EDCF varies from 0bits to 397bits and then to149bits. Therefore it is concluded that the results are better with EDCF.

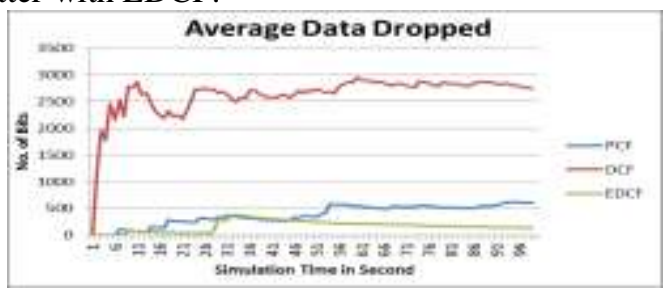

Fig 9: Average Wireless LAN Data Dropped 
In figure 10, It have been noticed that the Retransmission Attempts for PCF is 20Packets/Sec, DCF is 17 Packets/Sec and of EDCF is 30 Packets/Sec

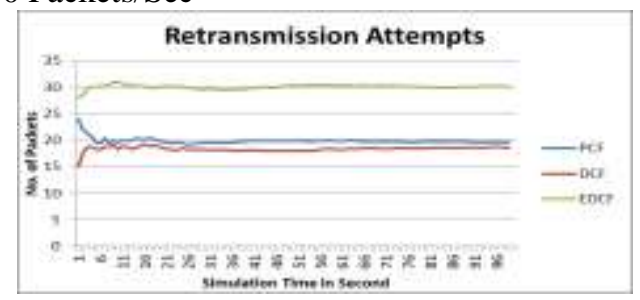

Fig 10: Average Wireless LAN Retransmission Attempts

In figure 11, It have been noticed that the scenario where PCF is used can handle load of the order of $1125 \mathrm{~kb} / \mathrm{sec}$. that the scenario where DCF is used can also handle load of the order of $1125 \mathrm{~kb} / \mathrm{sec}$. But with EDCF, It can handle Load upto $1319 \mathrm{~kb} / \mathrm{sec}$.

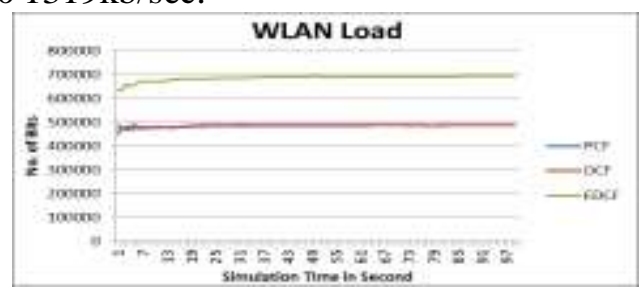

Fig 11: Average Wireless LAN Load

\section{CONCLUSION}

This paper investigates campus environment Hybrid Network. In this paper we have checked the performance of wireless Network using PCF, DCF \& EDCF co-ordination functions for different parameters like Channel Reservation, Data Traffic Received, Data Traffic Sent, Dropped Data Packet, Retransmission Attempts, and Load. Our investigations have revealed that Network having EDCF co-ordination functions is useful to improve the Quality of Service. Channel reservation with EDCF varies from 33ms to $34.7 \mathrm{~ms}$, with DCF and PCF it is $10 \mathrm{~ms}$. It has been noticed that the traffic sent for PCF varies from $659 \mathrm{~kb} / \mathrm{sec}$ to $677 \mathrm{~kb} / \mathrm{sec}$, DCF $662 \mathrm{~kb} / \mathrm{sec}$ to $675 \mathrm{~kb} / \mathrm{sec}$ and EDCF $613 \mathrm{~kb} / \mathrm{sec}$ to $685 \mathrm{~kb} / \mathrm{sec}$. It has been noticed that the Traffic Received for PCF varies from $27 \mathrm{~kb} / \mathrm{sec}$ to $10 \mathrm{~kb} / \mathrm{sec}$, DCF $139 \mathrm{~kb} / \mathrm{sec}$ to $149 \mathrm{~kb} / \mathrm{sec}$ and EDCF $269 \mathrm{~kb} / \mathrm{sec}$ to $279 \mathrm{~kb} / \mathrm{sec}$. it has been noticed that the Data Dropped for PCF varies from $0 \mathrm{bits} / \mathrm{sec}$ to $2761 \mathrm{bits} / \mathrm{sec}$, DCF $0 \mathrm{bits} / \mathrm{sec}$ to $625 \mathrm{bits} / \mathrm{sec}$ and with EDCF varies from 0bits to 397bits and then to149bits. It have been noticed that the Retransmission Attempts for PCF is 20Packets/Sec, DCF is 17 Packets/Sec and of EDCF is 30 Packets/Sec. It have been noticed that the scenario where PCF is used can handle load of the order of $1125 \mathrm{~kb} / \mathrm{sec}$. that the scenario where DCF is used can also handle load of the order of $1125 \mathrm{~kb} / \mathrm{sec}$. But with EDCF, It can handle Load up to $1319 \mathrm{~kb} / \mathrm{sec}$. Hence Wireless Network having EDCF co-ordination functions is very useful to improve the Quality of Service.

\section{References}

[1] Ashwini Dalvi, Pamukumar Swamy, B B Meshram, DCF Improvement for Satisfactory Throughput of 802.11 WLAN, International Journal on Computer Science and Engineering , 2011 Vol: 3 Issue: 7 ,2862-2868

[2] T Madhavi, G Sasi Bhushana Rao, M Rajan Babu, K Sridevi, Analysis of Throughput and Energy Efficiency in the IEEE 802.11 Wireless Local Area Networks using Constant backoff Window Algorithm, International Journal of Computer Applications, 2011,Vol: 26 Issue: $8,40-47$

[3] Vikram Jain, Sakshi Suhane, Performance Degradation of IEEE 802.11 MANET due to Heavy Increase and Heavy Decrease in Contention Window, International Journal of Computer Applications, 2011, Vol: 20 Issue: 6 ,26-32

[4] Ajay Dureja , Aman Dureja , Meha Khera, IEEE 802.11 Based MAC Improvements for MANET, International Journal of Computer Applications, 2011, Issue: 2 ,54-57

[5] Rama Krishna CHALlA, Saswat CHAKRABARTI, Debasish DATTA, An Improved Analytical Model for IEEE 802.11 Distributed Coordination Function under Finite Load, International Journal of Communications, Network and System Sciences, 2009, Vol: 02 Issue: $03,237-247$

[6] Yutae Lee, Min Young Chung, Tae-Jin Lee, Performance Analysis of IEEE 802.11 DCF under Nonsaturation Condition Mathematical Problems in Engineering, 2008

[7] Sunghyun Choi, Javier del Prado2, Sai Shankar N, Stefan Mangold, IEEE 802.11e Contention-Based Channel Access (EDCF) Performance Evaluation, vol.2, 2003, 1151 - 1156 